\title{
ESA Space Science Programme, Cosmic Vision 2015-2025, for astrophysics
}

\author{
Catherine Turon
}

GEPI - UMR CNRS 8111, Observatoire de Paris, Section de Meudon, F-92195 Meudon cedex, France email: catherine.turon@obspm.fr

\begin{abstract}
After a brief description of the ESA Science Programme, the long-term plan for Astrophysics is described, as well as possible strategies for its implementation.
\end{abstract}

Keywords. astrophysics, space, ESA

\section{Introduction}

The European Space Agency, ESA, is an inter-governmental organisation with a mission to provide and promote - for exclusively peaceful purposes - the exploitation of space science, research and technology, and space applications. The Science Programme is the only mandatory programme of ESA. With a budget of about $400 \mathrm{M} €$ per year, i.e., about $12 \%$ of the total ESA budget, it funds satellites, some (part of) payloads, satellite operation, data scientific validation and access to the data. The programme is chosen by the community, with long-term planning renewed every about 10 years. For over 30 years, ESA's space science projects have shown the scientific benefits of multi-nation cooperation.

Starting from April 2004, a new long term planning exercise was initiated by the ESA Science Programme. This plan is the third step in a decadal series. In 1983-1984, the first similar exercise, Horizon 2000 (Bonnet \& Bleeker 1984), replaced the previous à la carte style of mission selection. It is on the basis of this ambitious programme that Europe has developed $\mathrm{SOHO}$ and Cluster, landed on Titan with the Huygens probe early in 2005, built XMM-Newton and Integral, launched Mars Express, Rosetta and Venus Express. Two missions are still to come: Planck and Herschel. In 1994-95, the Horizon 2000-Plus plan (Bonnet \& Woltjer 1995) resulted in a series of high-profile missions which are now in preparation: Gaia, BepiColombo, and JWST, and also LISA in cooperation with NASA, and Solar Orbiter.

The numerous and enthousiast answers to the Call for Themes for Cosmic Vision 2015-2025 issued in April 2004 by the ESA Directorate of Science show the richness of new ideas coming from European scientists, and the potential for innovation open to industry.

On the basis of the many ideas proposed by the community, three main themes were identified by each of the ESA Science Working Groups. The three main themes identified by the Astronomy Working Group (Turon et al. 2005) were:

(a) other worlds and life in the Universe: detection, census and characterisation of exoplanets, search for extraterrestrial life, formation of stars and planetary systems;

(b) the Early Universe: investigating Dark Energy, probing inflation, observing the Universe taking shape; and

(c) the evolving violent Universe: matter under extreme conditions, black holes and galaxy evolution, supernovae and the life cycle of matter. 
These themes were debated during two meetings open to the community: a workshop in Paris in September 2004, and the 39th ESLAB Symposium, Trends in Space Science and Cosmic Vision 2020 (39th ESLAB Symposium), held at ESTEC in April 2005. In looking for synergies and coherence, four themes were finally selected by the Space Science Advisory Committee. They address four basic questions concerning the Universe and our place in it:

(a) What are the conditions for planetary formation and the emergence of life? Place the Solar System into the overall context of planetary formation, aiming at comparative planetology. The astrophysical aspects of this theme are two-fold: $(i)$ Map the birth of stars and planets by peering into the highly obscured cocoons where they form. Investigate which properties of the host stars are more favourable to the formation of planets;and (ii) Search for and image planets around stars other than the Sun, looking for biomarkers in their atmospheres. Direct detection of Earth-like planets. Physical and chemical characterization of their atmospheres for identification of unique biomarkers. Systematic census of terrestrial planets.Ultimate goal: image terrestrial planets.

(b) How does the Solar System work?

(c) What are the fundamental physical laws of the Universe?

(d) How did the Universe originate and what is it made of? This theme is essentially astrophysics, with strong connections to theme $(c)$ centred on the fundamental laws explaining the phenomena. This theme is divided in three parts: $(i)$ The early Universe: investigate the physical processes that lead to the inflationary phase in the early Universe during which a drastic expansion took place. Investigate the nature and origin of the Dark Energy that currently drives our Universe apart; (ii) The Universe taking shape: find the very first gravitationally bound structures assembled in the Universe (precursors to todays galaxies and clusters of galaxies) and trace their evolution to today; and (iii) The evolving violent Universe: formation and evolution of the super-massive black holes at galaxy centres, in relation to galaxy and star formation. Life cycle of matter in the Universe along its cosmic history.

These four themes are described in detail in Cosmic Vision 2015-2025, Space Science for Europe, 2015-2025 (Bignami et al. 2005).

\section{Implementation strategy}

To implement the major objectives of Cosmic Vision 2015-2025 while keeping some flexibility in the overall planning, successive slices of between 1 and $1.3 \mathrm{~B} €$ each will be considered. The first Call for Mission Proposals is expected to be issued in early 2007. The Call is planned to be fully open, i.e., with no a priori size restriction, but with clear different categories of cost and length of development for the proposed missions. Two categories are considered: proposals for medium size missions, with an overall cost to ESA smaller than $300 \mathrm{M} €$, and a first launch opportunity in the 2015-2018 timeframe; proposals for concepts of large size missions with an overall cost to ESA smaller than $650 \mathrm{M} €$. The concepts of missions selected in this category will serve for long term technological developments, with a first possible launch after 2020.

\section{References}

Bignami, G., Cargill, P., Schütz, B., \& Turon, C. 2005, ESA-BR 247, October 2005, ed. A. Wilson

Bonnet, R.-M., \& Bleeker, J. 1984, ESA-SP 1070, December 1984, eds. N. Longdon \& H. Olthof Bonnet, R.-M., \& Woltjer, L. 1994, ESA-SP 1180, November 1994, August 1995, ed. B. Battrick 39th ESLAB Symposium 2005, ESA-SP 588, eds. F. Favata, et al.

Turon, C., Done, C., Quirrenbach, A., et al. 2005, ESA-SP 588, eds. F. Favata, et al., p. 53 